\title{
The TactaPack: A Wireless Sensor/Actuator Package for Physical Therapy Applications
}

\author{
Robert W. Lindeman ${ }^{\star 1}$ \\ ${ }^{*}$ Dept. of Computer Science \\ Worcester Polytechnic Institute
}

\author{
Yasuyuki Yanagida ${ }^{\$ 2}$ \\ ${ }^{\dagger}$ Dept. of Information Engineering \\ Faculty of Science and Technology \\ Meijo University
}

\author{
Kenichi Hosaka ${ }^{\ddagger 3}$ \\ ${ }^{\ddagger}$ ATR International \\ Media Information \\ Science Laboratories
}

\author{
Shinji $A b e^{\S 4}$ \\ $\S$ ATR International \\ Intelligent Robotics and \\ Communication Laboratories
}

\section{Abstract}

In this paper, we present preliminary work we have done on designing the TactaPack, a wearable sensor/actuator device that uses a Bluetooth wireless connection to return sensor data to a host, and to receive commands to initiate expressive vibrotactile stimuli. We present our work in the context of a physical therapy application designed to provide more autonomy for patients when performing rehabilitative exercises. This assistive technology has the potential to reduce injuries during therapy due to improper patient joint movement, and decrease the workload of physical therapists, thereby reducing healthcare costs.

Though still in the early stages of design, we believe the TactaPack can be used to produce systems that are less cumbersome than current, wired solutions, and simplify the creation of high-level applications by offloading from the CPU to the device the process of sensing, testing against threshold values, and actuation.

CR Categories: H.5.2 [User Interfaces]: Haptic I/O; H.1.2 [User/Machine Systems]: Human factors; J.3 [Life and Medical Sciences]: Medical information sciences.

Keywords: Wireless sensor, actuator, virtual reality, physical therapy, vibrotactile.

\section{INTRODUCTION}

As part of our Networked Interaction Therapy (NIT) project [6], this paper explores the design, implementation, and testing of a physical therapy (PT) assistance tool. The overreaching goals of the multi-year NIT project include 1) increasing the independence of people dependent on medical care; 2) supporting "natural interaction" by sensing the patient's intention without the need for explicit user interaction; and 3) providing some relief for those in charge of caring for these individuals through network technology. The main parts of this system are an intention-detection mechanism, a stimulus-producing interface, and a community infrastructure platform. By observing the daily activities and behavior of brain-damaged subjects using vision technologies, her/his intention to communicate with others will be automatically detected, and s/he will be connected to a networked community. In order to lessen the burden of caretakers, visual, auditory, haptic, and olfactory senses are

\footnotetext{
1e-mail: gogo@wpi.edu

2e-mail: yanagida@ccmfs.meijo-u.ac.jp

en-mail: hosaka@atr.jp

e-mail: sabe@atr.jp
}

Symposium on Haptic Interfaces for

Virtual Environment and Teleoperator Systems 2006

March 25 - 26, Alexandria, Virginia, USA

1-4244-0226-3/06/\$20.00 @2006 IEEE stimulated so that the brain-damaged person can sustain attention while connected to a networked community. Development and evaluation of a networked community platform based on the sensory media interfaces described above is underway. This community consists of volunteers, caretakers, and healthcare professionals, all able to communicate with the patient using various communications technologies, depending on the nature of the task.

As an offshoot of this project, we are constructing a system to allow those recovering from joint replacement surgery to perform rehabilitative exercises using a novel interaction device called the TactaPack. Many developed nations are currently experiencing a "graying" of society, as the average age of citizens increases. This is due to such factors as the post-World-War-II "Baby Boom" and changing cultural norms, whereby couples are choosing to have fewer children than has traditionally been the case. This has led to the unfortunate situation of fewer people paying into public healthcare systems, combined with an ever-increasing number of people dependent on receiving healthcare benefits.

Using hip and knee replacement procedures as examples, 381,000 knee replacements and 193,000 hip replacements were performed in the US in 2002 (http://www.cdc.gov/nchs/). In Canada, there were 19,997 total-hip and 24,815 total-knee replacements performed on Canadian residents in 2001-2002, representing a seven-year increase of 39\%, up from 16,787 hip and 15,360 knee replacements in 1994-1995 [2].

Rehabilitation from joint replacement involves PT commencing almost immediately after surgery [14]. Repetitive exercises take the involved joint through increasing ranges of motion over a period of several months. In order to guard against injury prior to sufficient healing, specific motions of the involved joint should be avoided. For example, after hipreplacement surgery, patients should refrain from moving the knee of the involved leg towards the chest, or crossing the involved leg over the other leg (Figure 1).

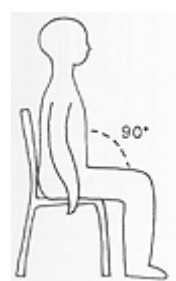

(a)

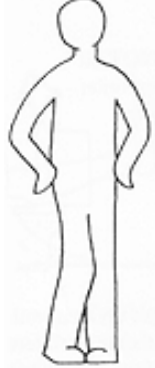

(b)

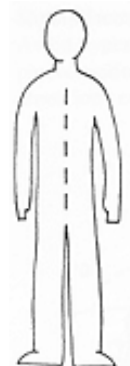

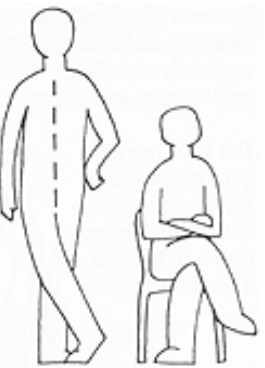

(c)
Figure 1: Examples of restricted movement subsequent to hip replacement surgery: (a) The involved hip should not be moved toward the chest (flexion) less than $90^{\circ}$; (b) the knee cap should not be turned inward when sitting, standing, or lying down; (c) the involved leg should not cross the body midline (toward the other leg) of the patient (adapted from [14]) 
Our aim is to warn patients during their exercise regimen when they are in danger of performing a movement that might result in injury to the joint recently operated on. In a calibration phase, sensor data is collected over a short period of time, during which the patient moves through the designated range of motion. Following calibration, the system monitors the patient's movements, and if the current movement violates the "safe" range of motion, a vibrational stimulus is delivered at the location of the violation to "nudge" the patient back into the proper movement range. The monitoring of patient movements and actuation of vibrational stimuli are performed using TactaPack devices we have developed. For motion following in rehabilitative exercises, the ability to control the intensity and timing of the vibration cues allows us to map the severity of the violation to the intensity of the vibration, and provides the vibration at the point of the violation. In this way, we are delivering cues that provide both temporal and spatial information.

\section{BACKGROUND}

The sense of touch is arguably the most complex of all the senses. This is partially due to several types of sensations all being attributed to this single "sense." Broadly speaking, our sense of touch can be divided into kinesthetic and cutaneous sub-senses. Kinesthetic stimulation maps roughly to forces being exerted on, and sensed by, mechanoreceptors in the joints, tendons, and muscles. For example, we feel the weight of a heavy object held in an upturned palm because the object weight exerts forces on the wrist, elbow, and shoulder joints, and we exert opposite forces to counter the weight. Proprioception, knowing where your limbs are without looking at them, is another example of a kinesthetic sense. Cutaneous stimuli, in contrast, are sensed through mechanoreceptors in the skin layers. There are several kinds of receptors, each allowing us to sense a different type of stimulus, such as thermal properties, vibration of varying frequencies, pressure, and pain. The sense of balance is also sometimes included as a cutaneous sense. The sense of touch is the only one where the entire system conducts both sensing and actuation, e.g., we maintain the equilibrium of an object held in our hands through a tight loop of sensing weight and exerting supportive forces.

In the current work, we focus on one of the more-common types of touch cues, vibrotactile cues. Several researchers have been working to develop the necessary technologies and techniques for deploying wearable, scalable, systems for delivering vibrotactile cues. We can identify several desirable attributes of such a system that would allow application developers to more-easily integrate such systems into their products. The first of these is expressiveness, meaning that such a system should allow for multiple tactors (devices that provide some form of tactile sensation) to be controlled, with multiple levels of vibration (e.g., intensity, frequency, amplitude), and with dependable timing, so that patterns could be reliably displayed. Second, the system should impose limited physical hindrance upon the user, meaning it should be easy to put on/take off the tactors and support hardware, and that movement should not be hindered by excessive cabling. Also, such a system should be easily scalable and reconfigurable, and the mapping of input data to stimulus output should be straightforward.

Several potential application domains have been identified, encompassing both virtual environments as well as the real world. Applications for virtual environments include providing force and torque information for molecular-docking tasks, creating a sense of motion using sensory saltation [13], and delivering cues when a user comes into contact with virtual objects [8], such as collision reaction vibrations in video games, rumble vibration for driving simulators, and high-frequency surface properties during active touching [3]. In terms of real-world applications, situation-awareness systems for pilots $[12,5]$ and road vehicle drivers, guidance systems for firefighters or blind individuals [1], motionfollowing systems for sports and fitness, such as for learning Tai Chi [4] or improving a golf swing (Innovative Sports Training http://www.innsport.com/), and non-verbal communication have been proposed.

The technologies for generating force-feedback (kinesthetic) stimuli are typically cumbersome, have limited range of motion, and are designed for special-purpose applications. On the other hand, these devices can typically generate strong forces and arrest user motion in a realistic manner. Tactile (cutaneous) devices typically generate weaker forces than force-feedback devices. Recent work has focused on the use of large numbers of inexpensive vibrating DC motors distributed over large areas of the body. These devices cannot arrest the motion of the user, but can provide a means for displaying information.

Many technological approaches to providing high-fidelity haptic cues require a significant portion of the apparatus to be mounted on the floor or fixed to a desk (e.g., PHANToM www.sensable.com), while others place a substantial weight burden on the user for untethered operation (e.g., CyberGrasp www.immersion.com). In general, systems which use low-cost vibration motors as a means of providing haptic cues trade high fidelity and precise control for simplicity, modest power consumption, and limited physical hindrance on the user.

\section{The TactaPack}

Over the past several years, we have been developing hardware, along with stimulus control techniques, for delivering vibrotactile cues for a range of applications in both real and virtual environments $[9,15,11]$. In a series of iterations, we have continued to increase the expressiveness of our delivery system, while decreasing the size and weight burdening the user. Our current version, the TactaPack, employs the widely-used Bluetooth standard as a communications vehicle, and incorporates an accelerometer to provide input, in addition to the tactor output.

\subsection{TactaPack Specifications}

Our TactaPack device builds on the framework first introduced for collecting movement data in a nursing environment [7]. The E-Nightingale project [6] strives to reduce the frequency of mistakes made in the healthcare industry through the use of unobtrusive monitoring technology, coupled with a patient information system. To this end, the project has developed devices that can collect large amounts of data from several types of sensors while only minimally influencing the normal working patterns of nurses. The TactaPack adds to the E-Nightingale hardware the ability to receive commands for triggering expressive vibrational stimuli from a host computer.

The TactaPack takes advantage of recent reductions in size of support chipsets for both processing and wireless communication. Also, new innovations in battery technology are used to provide long operating times. Finally, because we use a more-powerful processor than previous attempts, we also included high-level control in the firmware.

As shown in Figure 2 and Figure 3, the TactaPack uses a standard Bluetooth interface to control each unit. Because 
many laptop and desktop systems now come with built-in Bluetooth support, this makes system deployment very cost effective. The microprocessor is a Renesas Technology $\mathrm{H} 8 / 3687 \mathrm{~F}$ running at $7.3728 \mathrm{MHz}$. The version 1.2 , class 2 Bluetooth chipset we are using is part number WML-C29 from Mitsumi. A three-axis accelerometer, model number H48C from Hitachi Metals, is used as the sensor for this application. Each unit is powered by a rechargeable lithium-ion battery, part number IML-270530-2 from NEC Tokin, with a rating of 300 $\mathrm{mAh}$. The tactor is from Tokyo Parts Industrial Co., Ltd., Model No. FM37E, and has an operating voltage range of $2.5-3.8 \mathrm{~V}$ at $40 \mathrm{~mA}$. It has a frequency of $142 \mathrm{~Hz}$ at $3.0 \mathrm{~V}$, and a vibration quantity of $0.85 \mathrm{G}$.

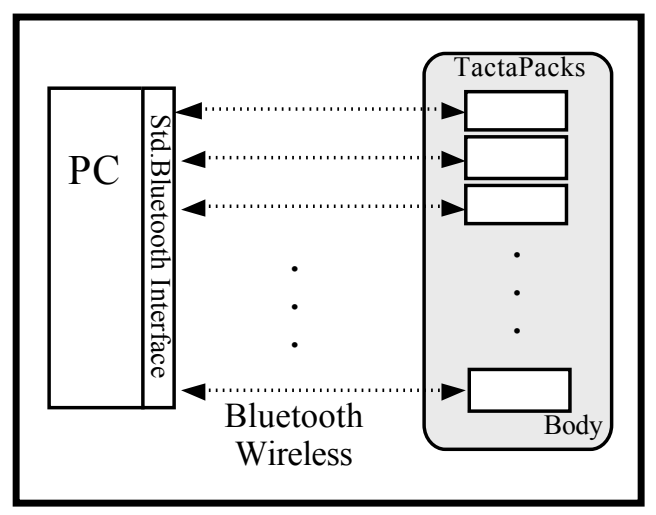

Figure 2: The TactaPack uses a standard Bluetooth connection to exchange commands and data with the host.

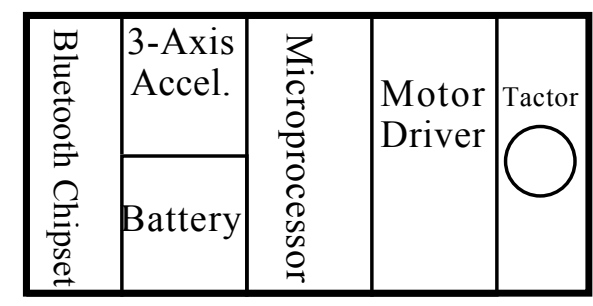

Figure 3: The TactaPack contains a Bluetooth chipset, a three-axis accelerometer, a microprocessor, motor driver, and a vibrotactile tactor.

In our testing, we were able to stream data from the sensor over Bluetooth continuously for eight hours before the battery needed re-charging. In addition, we tested battery life when vibration was used, and were able to pulse the tactor $\mathrm{ON}$ and OFF in half-second intervals for 1 hour and 20 minutes, while streaming sensor samples every five seconds. After this time, sensor return continued, but the battery could no longer support vibration. The physical, encased size of the current prototype is $58 \mathrm{~mm}(2.28 ") \times 35 \mathrm{~mm}\left(1.38^{\prime \prime}\right) \times 13.5 \mathrm{~mm}(0.53 ")$ $(\mathrm{H} \times \mathrm{W} \times \mathrm{D})$, and weighs $24.6 \mathrm{~g}$ (Figure 4).

In terms of interacting with the devices, an ASCII protocol allows for the host computer to collect data from the accelerometer and trigger vibration with varying intensity, depending on the application. The TactaPack has a local realtime clock in order to synchronize the acquired data. In general, the synchronization of distributed sensing data can be very important. However, data transmissions are sometimes interrupted and/or deferred due to radio conditions, especially in wireless networks. For the synchronization of the acquired sensing data, each TactaPack sends data with the local time stamp. In addition, a wake-up timer can be set to begin sensing at a specified time, relative to the on-board clock, and another can be set to vibrate at a certain time. If all TactaPacks are synchronized, they can be commanded to all vibrate at prespecified times, thereby providing support for fine-grained control, such as that required for producing apparent motion.

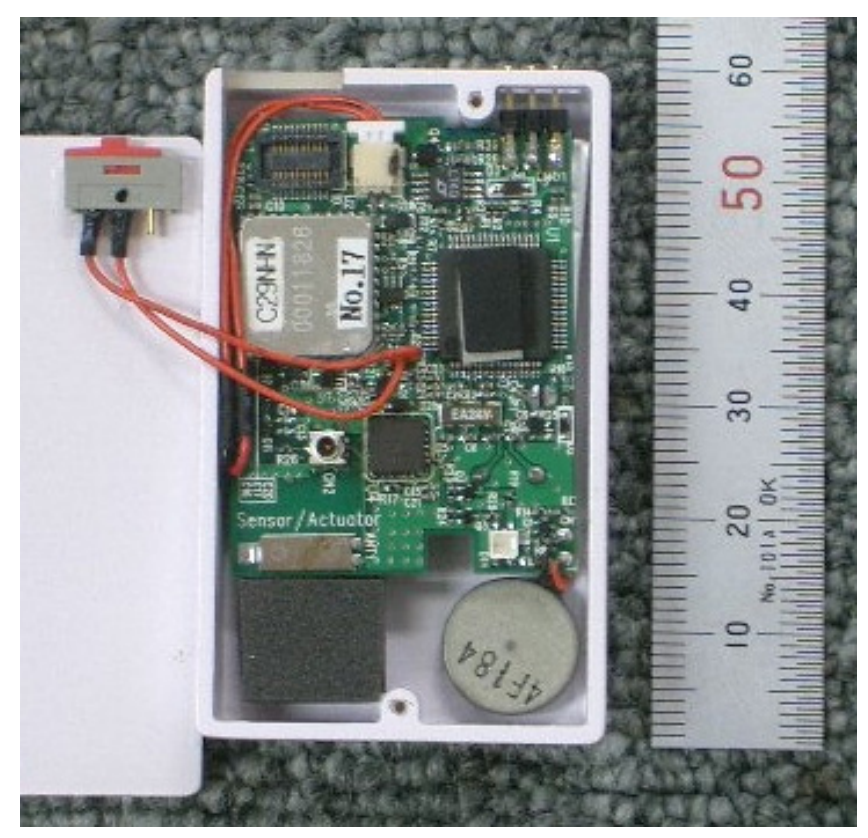

Figure 4: The TactaPack interior (with $\mathrm{mm}$ ruler).

The target application for this project is the use of sensor/actuator devices for monitoring patient movements in PT exercises following surgery, such as hip-replacement. To this end, higher-level commands can be sent to the TactaPack, so that it will vibrate when it detects movement that violates a certain constraint in any of the three axes. Both maximum and minimum constraints can be defined for each of the three axes. For instance, if a patient moves his/her hip outwards past a given threshold, the sensor will trigger a vibration to occur, "nudging" the patient back into a safe range.

\subsection{Wireless Connectivity}

To provide wireless network connectivity, we chose the Bluetooth standard for the following reasons. First, the use of Bluetooth is safe in many high-risk locations, such as hospitals. Some hospitals restrict the use of radio devices, such as mobile phones, because of the possibility they might interfere with other wireless equipment. However, because Bluetooth uses much less power than $802.11 \mathrm{~b}$, which uses the same ISM band, it can be used in many hospitals.

Second, the transmission reliability provided by Bluetooth improves our level of confidence in the delivery of commands, especially in environments where multiple devices must coexist. The Bluetooth specification includes support for simultaneous operation of multiple devices and covers many of the complex problems encountered in wireless networks, such as collisions and error detection/correction. Therefore, we expect reliable connectivity even if multiple devices are worn on several parts of a body at the same time. Also, because the low-level network functionality can be offloaded to the Bluetooth chipset, the microprocessor can dedicate itself to processes related to sensing and actuation. Finally, Bluetooth provides sufficient bandwidth to transmit fine-grained sensor 
data, approximately $400 \mathrm{kbps}$ in symmetric mode. Assuming that eight channels of 10-bit A/D data are acquired at $100 \mathrm{~Hz}$ and they are packed into 128 bits (with every 10-bits of data packed into 16 bits), the data rate is $12.8 \mathrm{kbps}$. Even if seven units are used simultaneously, requiring a bandwidth of 89.6 kbps, Bluetooth can successfully transmit the data, and still have a margin to transmit additional data. Moreover, Bluetooth is designed for short-range wireless networking, so the power consumption is relatively low, which is suitable for our use of the TactaPack.

The Bluetooth specification only allows each device to communicate with seven other active devices at a time. However, by using several Bluetooth controllers, we can support a much larger number of TactaPacks. And because Bluetooth control devices are fairly inexpensive (less than US\$15), this provides cost-effective scalability.

\section{Preliminary Physical Therapy System}

A screen capture of a PT session is shown in Figure 5. Most of these values will be fixed once the system is deployed, but are accessible during our prototyping stage. The vibration parameters are used to define the properties of the vibration cues that should be delivered. These include the delay before the onset of the vibration signal, as well as the period and duty cycle of the signal. The sensing parameters are used to control how the accelerometer samples are taken. These include the time between successive samples, as well as how many samples should be averaged before being used to compare against threshold values. Calibration parameters are used to set the timing for the rest and sample steps (see below). The threshold values for the accelerometers, and the number of repetitions for the PT session can also be set.
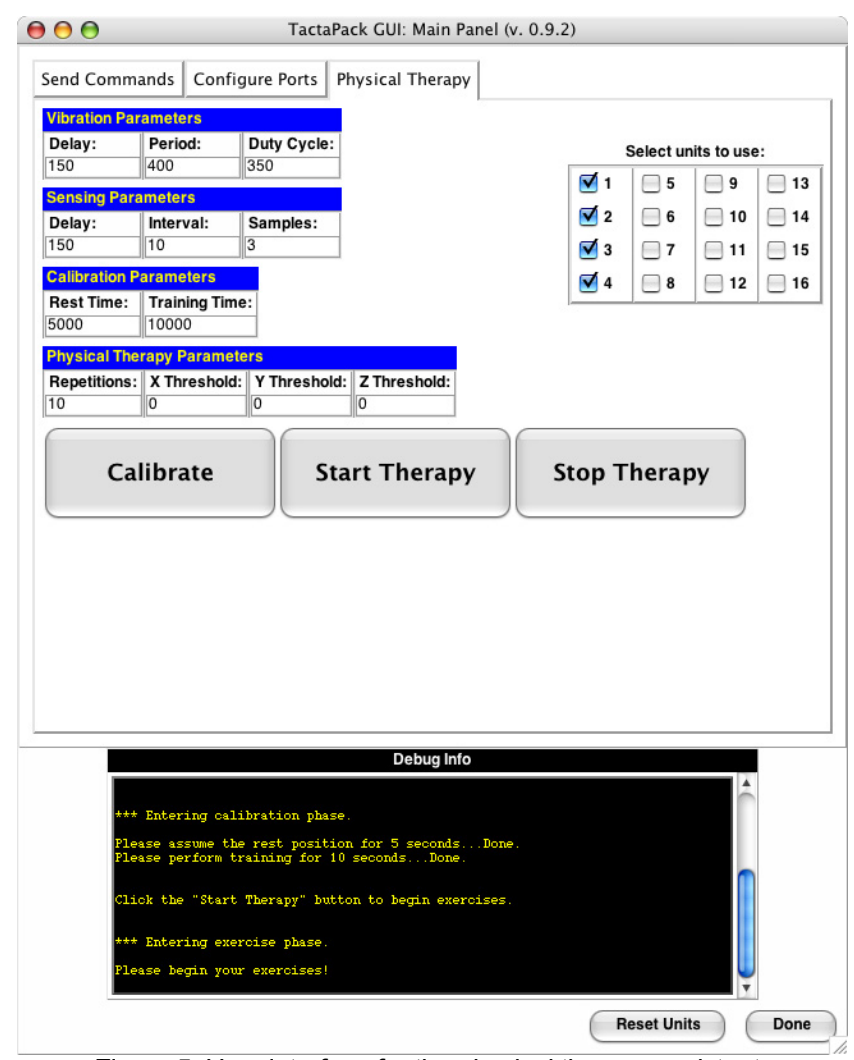

Figure 5: User interface for the physical therapy assistant.

\subsection{Sensing}

Currently, the accelerometer in each TactaPack only measures the momentary tilt of the sensor with respect to the gravity vector. The three-axis accelerometer measures this tilt for each axis independently, and the microprocessor computes a moving average over the number of samples specified in the interface, compares them to the threshold values collected during calibration, and, if necessary, triggers the tactor. The interval between samples (in $\mathrm{ms}$ ) is specified in the user interface. The processing power of the microprocessor limits the minimum value for this interval to be about $10 \mathrm{~ms}$. By setting the number of samples to three, the delay before triggering vibration is on the order of $50 \mathrm{~ms}$, which is sufficient, as the spin-up (or spin-down) time for the tactor to reach maximum vibration is on the order of $110 \mathrm{~ms}$. Empirical study will help to better understand these parameters, but we feel that the speed of the motion typical for this sort of therapy will allow us to react in time. It should be noted that we are not accumulating error due to drift (common when using these types of sensors), as we are using the momentary tilt, and not the accumulated acceleration.

\subsection{Actuation}

The vibration technology used in the TactaPack is fairly simple. The speed of a DC motor with an eccentric mass is controlled by varying the duty cycle of a pulse-width modulation (PWM) signal. As the microprocessor has built-in support for generating a PWM signal, this approach is efficient in terms of the number of microprocessor instructions involved. PWM approaches also typically use less power than approaches that vary the actual voltage sent to the motor. As mentioned above, there is about a $110-\mathrm{ms}$ delay from the time the microprocessor begins sending voltage to the tactor until the desired vibration level is achieved. While voice-coil-type or piezo-type tactors have much smaller latencies, the driving current and circuitry needed to control their vibration is more complex. We have traded low-latency and more-precise stimulus signal control for reduced complexity and longer battery life.

\subsection{Therapy Session}

Each PT session consists of two phases, a training phase (I) and a therapy phase (II). The patient first places the desired number of TactaPack units, typically two to four, on the limb segments around the involved joint. For the first few times a patient performs therapy after the operation, the physical therapist would position the TactaPack devices on the patient. As the patient gains experience with the routine, s/he could don and doff the equipment independently. During calibration, the patient is instructed to keep the involved joint in a rest position for a period of time (typically five seconds) while the system collects data to recognize the rest position in Phase II. Following this, the patient is instructed to perform their PT regimen, aided by the therapist, for some time (ten seconds). During this step, the maximum and minimum values for each axis of the three-axis accelerometer are collected.

Once calibration is complete, the patient can begin the actual therapy phase. In Phase II, the system uses the restposition data from Phase I to sense when the patient has completed a repetition. If the patient moves the involved limb out of the rest position, and then returns, one repetition is counted. The movement data collected during Phase I is used to monitor the motions being carried out by the user. If a TactaPack sensor detects a value that is greater than the 
maximum values, or less than the minimum values, the tactor for that TactaPack is triggered, thereby delivering a "nudge" to the patient at the point of the violation. We plan to add hysteresis values that can be set to smooth out sensor noise and actuator flip-flopping.

One approach to designing a wireless system for PT would be to have sensors and actuators positioned on the patient, and then to use wireless communication to send sensor data to the host, check for constraint violations, and then to send actuation commands when necessary. This approach would allow the sensor and actuation units to be very small, but would incur a (possibly-variable) communications delay. This delay creates the potential for the system to respond too late to dangerous movements, leading to patient injury.

The TactaPack provides support for closed-loop control by allowing the application to define values that should be used as thresholds for triggering vibration cues, and letting the TactaPack handle the sensing, constraint checking, and actuation. Additionally, the values collected by the accelerometer can be sent back to the host application for further processing or archiving.

\section{COnclusions And Future Work}

The main goal of our multi-year research project is to increase the independence of those who are reliant on caregivers in order to carry out their daily activities. Within this context, we have been working to design a system to assist patients in performing their required rehabilitative exercises, thereby reducing their reliance on physical therapists. This type of system could potentially decrease healthcare costs. We are currently in the process of refining our design, and locating physical therapists to help design and refine our system, and appropriate centers where we can perform trials of the system.

There are several aspects of our current system that we are refining. Currently, we are only performing simple constraint checking. This can lead to falsely triggering the vibration due to accelerometer noise. We are interested in implementing more-sophisticated pattern-matching routines commonly employed in other fields, such as speech recognition, in order to reduce false positives.

Another way to improve accuracy is to use multiple TactaPacks in concert. Currently, each TactaPack monitors and actuates in isolation. Coordinated sensing could improve the accuracy by using higher-level measures of movement, such as how much one TactaPack has moved in relation to another.

Other applications for this type of system are also being considered. As the system continues to be miniaturized, the TactaPacks could be used while the patient sleeps to monitor patient movements, and deliver an alert when there is risk, for example, to avoid bedsores, or to guard against movements that might cause injury to a healing joint. In the field of virtual reality, where encumbrances are a constant issue, these devices could be used in virtual contact situations. We have incorporated them into our next-generation TactaVest [10], to provide more flexibility in tactor placement, as well as making tactor removal easier for cleaning the garment.

We are also looking at using a more-powerful microcontroller. By incorporating more-sophisticated control on the TactaPack, we could further offload work from the host, distributing the workload more efficiently.

Our design also provides for other types of sensors (and/or actuators) to be supported, with little change to the hardware. We have considered connecting temperature sensors to assess user status, as well as simple buttons to allow for intentional user input to be realized. In addition, the remaining capacity of the Bluetooth connection allows us to transmit additional data using the same connection. One idea is to send voice data captured by an attached microphone. This would allow us to decouple the sensing and recording aspects for use in the ENightingale project, further reducing the equipment deployed on the nurses.

\section{ACKNOWLEDGMENTS}

This research was supported in part by the project "Networked interaction therapy" with funding from the National Institute of Information and Communications Technology, Japan.

\section{REFERENCES}

[1] T. Amemiya, J. Yamashita, K. Hirota and M. Hirose, "Virtual Leading Blocks for the Deaf-Blind: A Real-Time Way-Finder by Verbal-Nonverbal Hybrid Interface and High-Density RFID Tag Space," Proc. of IEEE Virtual Reality 2004, pp. 165-172.

[2] Canadian Institute for Health Information, Canadian Joint Replacement Registry 2004 Report, Total Hip and Total Knee Replacements in Canada March 9, 2004, ISBN 1-55392-352-9 (Paper), ISBN 1-55392-368-5 (PDF)

[3] S. Choi and H.Z. Tan, "An experimental study of perceived instability during haptic texture rendering: Effects of collision detection algorithm," Proc. of the 11th Int'l. Symp. on Haptic Interfaces for Virtual Environment and Teleoperator Systems, 2003, pp. 197-204.

[4] P.T. Chua, R. Crivella, B. Daly, N. Hu, R. Schaaf, D. Ventura, T. Camill, J. Hodgins and R. Pausch, "Training for physical tasks in virtual environments: Tai Chi." Proc. of IEEE Virtual Reality 2003, pp. 87-94.

[5] J.B.F. van Erp and H.A.H.C. van Veen, "A Multi-Purpose Tactile Vest for Astronauts in the International Space Station," Proc. of Eurohaptics 2003, pp. 405-408.

[6] Intelligent Robotics and Communication Labs. [Web document]. ATR International, 2001: [2005]. http://www.irc.atr.jp/projecte.html.

[7] N. Kuwahara, H. Noma, N. Tetsutani, K. Kogure, N. Hagita and H. Iseki, "Wearable Auto-Event-Recording System for Medical Nursing," Proc. of Human-Computer Interaction INTERACT'03, 2003, pp. 805-808.

[8] R.W. Lindeman, J.N. Templeman, J.L. Sibert and J.R. Cutler, "Handling of Virtual Contact in Immersive Virtual Environments: Beyond Visuals," Virtual Reality, 6(3), 2002, pp. 130-139.

[9] R.W. Lindeman and J.R. Cutler, "Controller Design for a Wearable, Near-Field Haptic Display," Proc. of the 11th Symp. on Haptic Interfaces for Virtual Environment and Teleoperator Systems, 2003, pp. 397-403.

[10] R.W. Lindeman, R. Page, Y. Yanagida and J.L. Sibert, "Towards Full-Body Haptic Feedback: The Design and Deployment of a Spatialized Vibrotactile Feedback System," Proc. of ACM Virtual Reality Software and Technology (VRST) 2004, Nov. 10-12, 2004, Hong Kong, China, pp. 146-149.

[11] R.W. Lindeman, J.L. Sibert, E. Mendez-Mendez, S. Patil and D. Phifer, "Effectiveness of Directional Vibrotactile Cuing on a Building-Clearing Task," Proc. of ACM CHI 2005, Apr. 2-7, 2005, Portland, Oregon, USA, pp. 271-280.

[12]A. Rupert "An instrumentation solution for reducing spatial disorientation mishaps," IEEE Eng. in Med. and Bio. 2000, pp. 7180.

[13] H. Tan, A. Lim and R. Traylor, "A psychophysical study of sensory saltation with an open response paradigm," Proc. of the 9th Int'l Symp. on Haptic Interfaces for Virtual Environment and Teleoperator Sys., 2000, pp. 1109-1115.

[14] Total Hip Replacement: A Guide for Patients. [Web document]. The Univ. of Iowa: Virtual Hospital, 1986: [2005]. http://www.vh.org/adult/patient/orthopaedics/hipreplace/

[15] Y. Yanagida, M. Kakita, R.W. Lindeman, Y. Kume and N. Tetsutani, "Vibrotactile Letter Reading Using a Low-Resolution Tactor Array," Proc. of the 12th Symp. on Haptic Interfaces for Virtual Environment and Teleoperator Systems (2004), pp. 400-406. 